\title{
LV-PP-3-1
}

\section{Predictive implication for liver regeneration by transient elastography in patients with major hepatectomy of perihilar cholangiocarcinoma}

\author{
Dakyum SHIN, Jae Hoon LEE*
}

Division of Hepatobiliary and Pancreatic Surgery, Department of Surgery, Asan Medical Center, Seoul, Korea

Introduction: There are many studies regarding postoperative liver regeneration after major hepatectomy related to posthepatectomy liver failure (PHLF). However, there are no study which focused on liver fibrosis measured by transient elastography and liver regeneration. Therefore, we aimed to evaluate the correlation between transient elastography and liver regeneration in patients who underwent surgery for perihilar cholangiocarcinoma (PHCC).

Methods: Patients who underwent major hepatectomy for PHCC with curative intent between 2016 and 2020 were included in the study. Patients were divided into groups by the value of liver stiffness measured by transient liver elastography and the cutoff values for severity of fibrosis were derived from the study by Tsochatzis et al. Liver volumes were compared between preoperative and postoperative day 5 CT scan.

Results: Of the 45 patients who underwent surgery for PHCC, F1 group was consisted of 23 (51.1\%) patients, F2 group 10 (22.2\%), F3 group 9 (20.0\%), and F4 group 3 (6.7\%) patients. The patients were divided into 2 groups, F1 and F2 as Group A and F3 and F4 as Group B. The ICG R 15 value was $14.7 \pm 6.6$ in Group A and $20.3 \pm 9.9$ in Group B $(p=0.034)$. The mean value of liver volume increase rate of each group was $42.3 \% \pm 30.9 \%$ in Group A, and $37.4 \% \pm 24.6 \%$ in Group B ( $p=0.622)$.

Conclusions: This study suggests that liver fibrosis has a tendency to be related with less liver regeneration but without clinical significance. However, transient elastography could be used as one of the useful predictor reference for liver regeneration related to PHLF. 